\title{
A Dynamic Dextral-Sinistral Model for the Structure and Evolution of Prominence Magnetic Fields
}

\author{
E. R. Priest
}

Mathematical and Computational Sciences Department, St. Andrews University, St. Andrews, KY16 9SS, Scotland, U.K.

Abstract. We propose the following coherent model for prominences.

(i) They are composed of many fine threads whose mass we suggest has two kinds of origin. Some of the mass may be lifted from the upper photosphere in response to cancellation of magnetic flux in small flux tubes. If this rate of mass supply is slow then the mass needs to be supported, but if the mass supply rate is as large as the natural rate of draining then no support is necessary. However, much of the mass may not need to be supplied or supported at all, if it comprises dynamic threads of cool plasma that are in pressure balance with their hot surroundings and are continually forming from the corona and heating up again in response to a localised heating mechanism.

(ii) The overall structure is of a large-scale flux tube along the polarity inversion line with an overlying arcade that links the network on either side of the filament channel. The structure of the channel and of barbs is explained by the distribution of flux sources along the channels, with majority polarity producing plagettes and minority polarity the barbs.

(iii) The chirality of polar-crown prominences is suggested to be caused by a combination of subsurface flows, flux emergence and reconnection. By contrast, two generic types of low-latitude filament, namely those associated with unipolar flux tongues and with the emergence of new active regions near to remnant regions, are a natural consequence of nonuniform flux distribution and converging flux.

\section{Introduction}

Prominences owe their birth, maintenance and death to the magnetic field, so I plan here to give an overview of the role of the magnetic field in this structure and evolution. At the IAU prominence colloquium in Hvar eight years ago there were several paradigms about prominences, namely:

(A) Formation is by radiative instability when the length is too long;

(B) The structure is a sheared force-free field of Normal or Inverse type;

(C) Prominence material is basically static, supported in a magnetic dip;

(D) The eruption occurs when the twist or shear are too great.

How has our understanding developed since then? In attempting to answer that question I shall consider the local thread structure (Section 2), the largescale structure (Section 3) and the chirality (Section 4). 


\section{To Support or Not to Support - A Dynamical Model}

That is a key question about the nature of prominences. The assumption that support is necessary and that a magnetic dip is necessary to provide such support has dominated the theory of prominences for many years. But is support necessary? If a prominence consists of a static slab of uniform plasma, then the answer is clearly yes, since the free-fall speed from a height of, say $30 \mathrm{Mm}$, is about $100 \mathrm{~km} \mathrm{~s}^{-1}$, whereas most of the observed plasma is moving much more slowly. Furthermore, hydrostatic equilibrium along an inclined field line would produce an exponential fall-off of pressure with height $\left(p=p_{0} \exp (-z / H)\right)$ with a scale-height $(H)$ of typically $200 \mathrm{~km}$ at prominence temperatures, whereas such a rapid fall-off is not observed in prominences. A similar problem would arise in fibril plasma if a cool fibril were in pressure balance with its hot environment and its vertical thickness were more than about $200 \mathrm{~km}$ since the pressure would decrease substantially across it.

The most effective way of supporting plasma is in a magnetic dip. Such a dip along the length of a prominence is unlikely since the magnetic field would need to be exceptionally flat: a dip of only $400 \mathrm{~km}$ along the $100 \mathrm{Mm}$ length of a prominence would, for instance, make the central pressure ten times the end pressure. The general assumption, therefore, has been that the magnetic field lines cross the prominence at a narrow angle and that they have a very shallow dip, as in a weakly twisted flux tube. Thus, for example, a ratio of vertical to horizontal field in a plane perpendicular to the polarity inversion line of only 0.05 would naturally support a prominence of width $2 \mathrm{H} / 0.05$, or about $6 \mathrm{Mm}$, as observed. Thus the natural explanation of the narrow width of a prominence has been a good feature of a twisted flux tube model.

However, how fast would plasma drain out of a flux tube along a field line inclined at $\theta$ to the horizontal? Accelerating from rest under gravity alone a plasma element would fall a vertical distance $h=s \sin \theta$ in a time $t_{f}=\sqrt{ }\left(2 h / g \sin ^{2} \theta\right)$. For example, if the vertical distance $(h)$ were $10 \mathrm{Mm}$, say, and the inclination angle were $5^{\circ}$, the fall time $\left(t_{f}\right)$ would be about an hour, and if the angle were $1^{\circ}$ it would be about four hours. Other effects such as pressure gradients and viscous forces could increase these times substantially. If the rate of mass supply is low then the plasma needs to be supported in a prominence. However, the above estimate suggests that in a dynamic model support is not necessary in two distinct variants. The first is if the plasma is continually brought up from the chromosphere and replenished rapidly enough, and the second is if cool plasma threads do not live much longer than a few hours but simply represent coronal plasma that is continually condensing and reheating.

\subsection{Mass Supply in Response to Flux Cancellation}

Flux cancellation is a common occurrence under prominences at several locations along a filament channel. We have therefore suggested in our earlier formulation of a chiral dextral-sinistral model (Priest et al. 1996) that such sites represent regions where field lines are reconnecting and carrying mass up into the corona. This represents a natural way of replenishing mass in a prominence. Provided such a rate of mass supply is efficient enough, it can balance the rate of draining and remove the need for support. By means of a simple model we have calculated 
the height to which the captured mass is raised and the way the mass spreads out along a field line as it moves upward. These, together with the resulting plasma density, all compare favourably with what is needed in a prominence.

\subsection{Dynamic Recycling of Thread Plasma}

Instead suppose a prominence consists of threads that are in pressure balance with their surroundings and are continuously forming from coronal plasma by radiative condensation and are then heating up again in a random manner in response to a sporadic heating process. This can either take place in a region of horizontal or dipped field or, if the time for draining exceeds the time between heating bursts, in an inclined field.

Prominence threads are typically $200-300 \mathrm{~km}$ wide and $5000 \mathrm{~km}$ long and have lifetimes of about $8 \mathrm{~min}$ or even as short as $1 \mathrm{~min}$ (Engvold 1989). Their temperatures and densities are typically $8000 \mathrm{~K}$ and $10^{16} \mathrm{~m}^{-3}$ and so could be in pressure balance with an ambient coronal plasma of $10^{6} \mathrm{~K}$ and $10^{14} \mathrm{~m}^{-3}$. The magnetic field is not thought to vary substantially across them (Leroy 1989).

Let us consider an elementary cooling-heating process in a fibril along the magnetic field. Suppose we start with uniform coronal plasma at temperature $T_{h}$

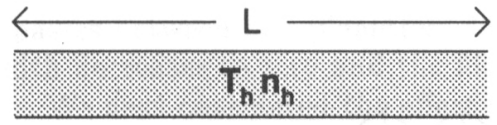

(a)

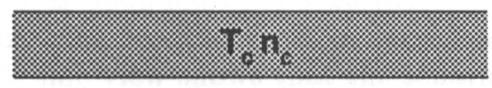

(c)

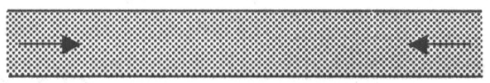

(b)

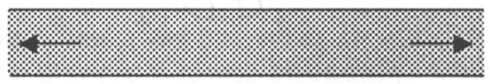

(d)

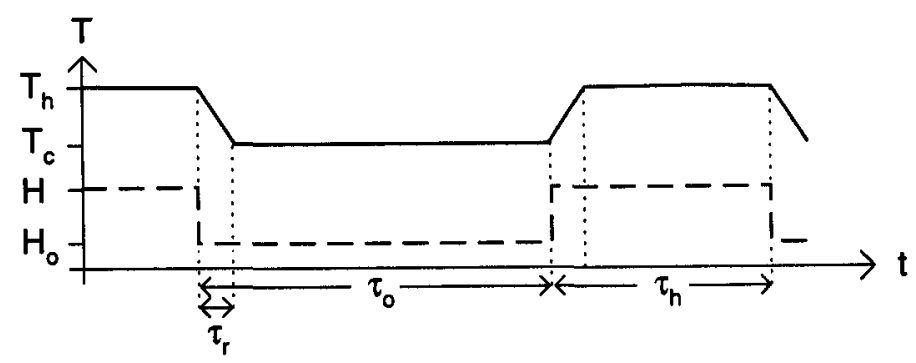

Figure 1. (a) - (d) An elementary formation-dissolution process for a fibril of temperature $T_{c}$ that forms from corona of temperature $T_{h}$. (e) A schematic of the heating (dashed) and temperature (solid) profiles.

and density $n_{h}$ (Figure 1a) in equilibrium under a balance between say heating of strength, $H$, and conduction over a long length-scale, $L_{e}$. Suppose next that the heating over a length, $L$, is switched off and the plasma cools down (Figure 1b) 
with plasma flowing into the region of radiative condensation. Eventually after a response time $\tau_{r}$, say, a new cool fibril equilibrium is set up at temperature, $T_{c}$, and density, $n_{c}$ (Figure 1c), which is maintained for a time, $\tau_{0}$, after the heating was switched off. Then suppose the heating is switched on again so that for a further time, $\tau_{r}$, the plasma heats up and expands along the field (Figure 1d) until a new hot equilibrium is established once more for a time $\tau_{h}$, say.

The whole process will take place at uniform pressure if the time-scales are much longer than the sound-travel time $\left(\tau_{s}=L / c_{s}\right)$ along the fibril. The responsive time $\left(\tau_{r}\right)$ is the minimum of the radiative time $\left(\tau_{r a d}\right)$ and the conduction time $\left(\tau_{\text {cond }}\right)$, provided this is longer than the time $\left(\tau_{s}\right)$ it takes to switch off or on the heating process. We suggest that this elementary process is repeated in neighbouring flux tubes due to randomly distributed impulsive heating.

\section{To Twist or Not to Twist - A Global Flux Tube}

There are three main elements to the global field around a prominence in our model, namely the polarity inversion line (PIL), a large-scale horizontal flux tube aligned along the PIL (Figure 2) and an overlying magnetic arcade, anchored in the network elements both sides of the PIL.

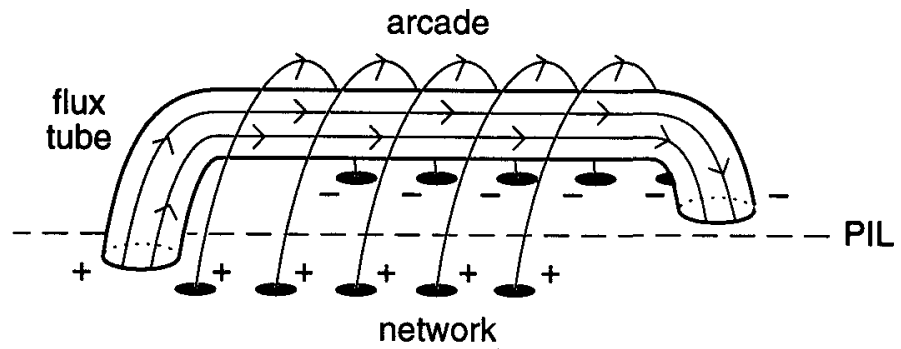

Figure 2. Global field structure for a dextral prominence with a large flux tube along the polarity inversion line underneath the arcade associated with the network on either side of it.

A key question for the global structure is whether the flux tube is always twisted or not. Often such twist is not observed, but of course absence of evidence is not the same as evidence of absence, since the structures you see in $\mathrm{H} \alpha$, for instance, do not necessarily always indicate the magnetic structure and a twisted flux tube filled with uniform plasma would show up as uniform untwisted tube. Thus, a weak twist may still be present when it is not observed. Furthermore, such a twist is reasonable on theoretical grounds: it naturally explains the narrow width of a prominence and, if reconnections are taking place, the magnetic helicity will be conserved and will relentlessly build up (unless it cancels with opposite helicity). Furthermore, a helical flux tube is naturally created by reconnection when the opposite-polarity footpoints of two neighbouring loops approach the polarity inversion line and cancel (van Ballegooijen and Martens 1989). Thus some twist is natural, but the question still arises whether twist is essential to the existence of a prominence or not. 
Flux tube models naturally stress the component of the field along the prominence. Hood and Priest (1979) suggested that an active-region filament is a flux tube filled by radiative condensation with cool plasma. At the Hvar colloquium we proposed a global flux tube model (Priest et al. 1989) which has since been developed and modified by several authors (e.g., Antiochos and Klimchuk, 1993, Low, 1991, Rust and Kumar, 1995).

\subsection{Structure of the Filament Channel}

We adopt the basic structure of the prominence environment shown in Figure 2 with a region of strong horizontal flux along the polarity inversion zone between the opposite polarity network. But what creates the detailed structure in filaments and filament channels, such as the kinks and gaps, that are often seen? We suggest they are a natural consequence of the location and strength of individual flux sources along the channel which show up as plagettes in $\mathrm{H} \alpha$ and have the same polarity as the network. We have modelled such a channel by superposing the field of a flux tube and that of plagette sources (Mackay and Priest 1996) and calculate the effect of the plagette flux $(f)$ on the path of the filament and the dimensions of the plagette magnetosphere. As $f$ increases, the kinking of the filament increases in amplitude and the width of the filament, as it passes between two plagettes, decreases. Eventually, when $f$ is too large the width decreases to zero and the filament breaks into two parts.

We next considered the effect of neighbouring network flux on the filament and its channel (Mackay and Priest 1996). The simplest model consists of a pair of line sources (the network sources either side of the polarity inversion line) together with a source $(f)$ and a sink $(-f)$ of the filament channel flux. The view from above shows that the filament channel flux linking the source to the sink is bounded by a separatrix (dashed) which separates the filament channel flux from flux that links either the source or the sink to the network. In addition, the side view shows that the separatrix surface possess a lower boundary, which may explain the base of the prominence; below it, flux joins the two networks. Furthermore, the upper boundary may account for the top of the prominence or the coronal cavity; above it again flux links the two networks.

This idea has been used to model a particular filament forming between a remnant active region and a newly emerging region (Figure 3a). The magnetic structure deduced from photospheric magnetograms shows that the filament channel is a very narrow structure enclosed in a separatrix which shields the filament from the surrounding magnetic field (Figure $3 \mathrm{~b}$ ).

\subsection{Structure of Barbs}

One of the intriguing puzzles about prominences is the presence of barbs or feet, which are transient features with a lifetime of about eight hours, comparable with the lifetime of intra-network magnetic fragments. The key question to begin with, is to ask whether a barb represents a magnetic field line (Figure 4a), along which plasma can flow freely, or whether it represents a region of horizontal field that supports plasma (Figure 4b). The elegant model of Aulanier and Demoulin (1998) is of the second type and is very appealing, although the short lifetime of barbs suggests that support may not be necessary and the observed frequent downflow suggests that our first model may be preferable. 

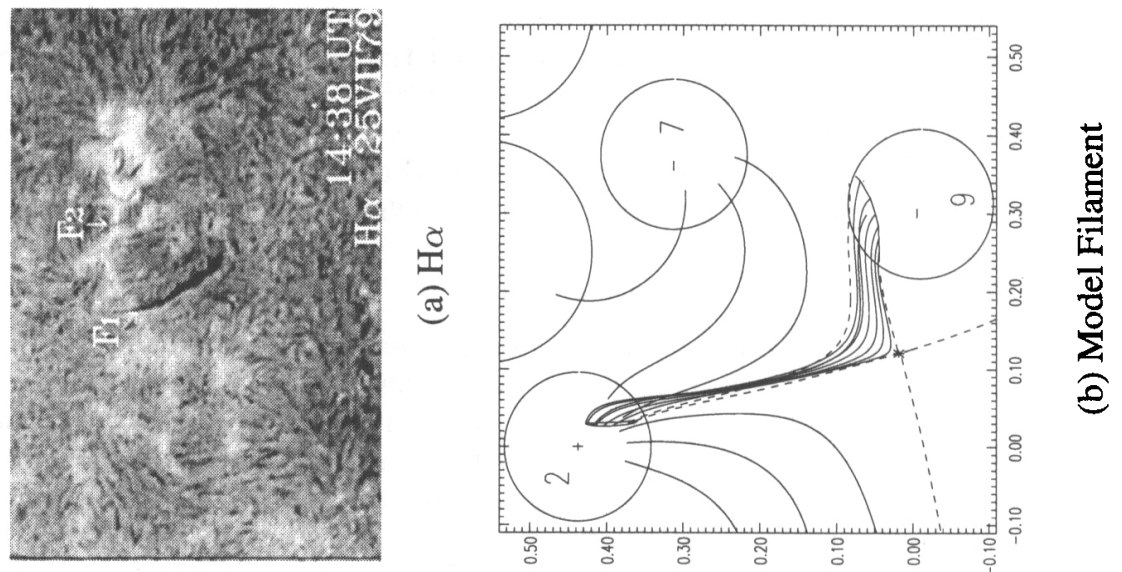

Figure 3. (a) A newly formed filament (b) a close-up of the magnetic structure near the filament (Mackay et al. 1997).

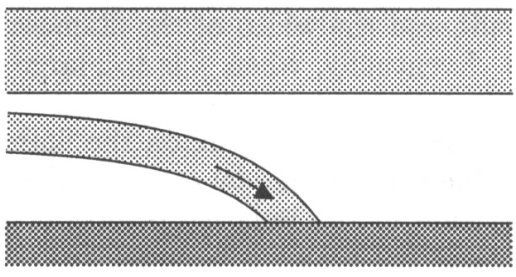

(a)

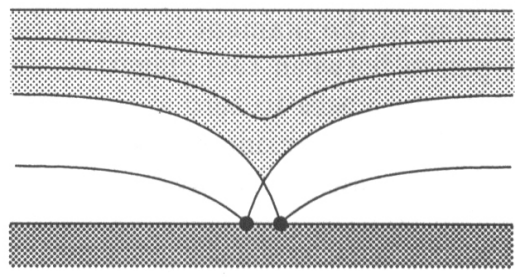

(b)

Figure 4. Two possible configurations for a barb.

The second important question is: why do barbs only appear with the correct sense, i.e., dextral prominences have right-bearing barbs rather than left-bearing ones? We suggest that this is because the new bipoles tend to emerge below or close to the prominence with a preferred orientation due to the direction of the global subsurface flux (Figure 5). Thus when a new bipole emerges with that orientation, it will then reconnect and produce a dextral barb in the northern hemisphere and a sinistral barb in the southern hemisphere.

\section{Chirality}

One of the most important discoveries to my mind over the past eight years is that of chiral structure by Martin et al. (1994), the fact that all prominences are either dextral or sinistral and that most quiescent prominences in the northern hemisphere are dextral whereas those in the southern hemisphere are sinistral. So, what is the cause of the chiral structure? I would like to suggest that it is a combination of the convergence of flux towards the polarity inversion line with three distinct effects:

I. Surface flows such as differential rotation, meridional flow and active-region 


\section{DEXTRAL BARB CREATION by FLUX EMERGENCE}
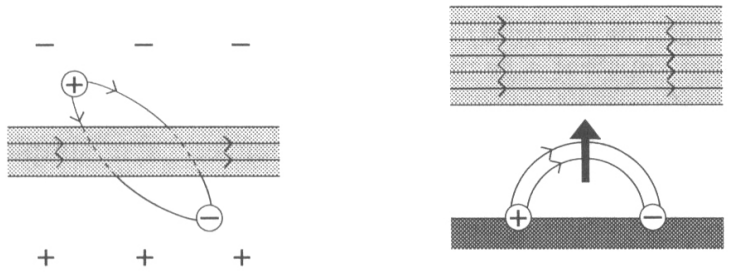

(a) flux emergence
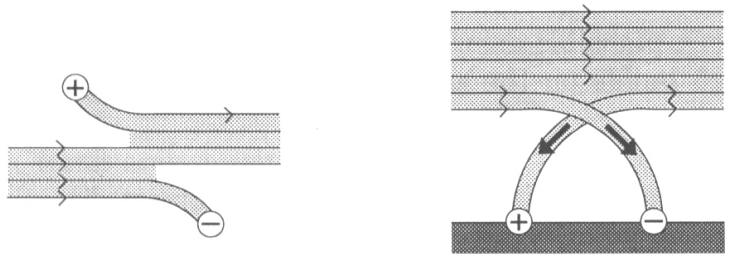

(b) reconnection with the prominence

Figure 5. (a) The emergence of new flux near a filament (b) to give a barb, as viewed from above (left) or the side (right).

evolution, which shear the footpoints of coronal fields;

II. Subsurface evolution and emergence;

III. Generic flux distributions due to the locations of magnetic sources, which produce a potential magnetic field not necessarily normal to the inversion line.

Let us now consider the effect of these three processes to see whether we can explain different types of prominence.

\subsection{Polar-Crown Prominences}

Differential rotation acting on a north-south polarity inversion line can produce the correct chirality (van Ballegooijen and Martens 1990). However, when it acts on the east-west polarity inversion line that is typical of the polar crown it produces loops that have exactly the wrong chirality for prominences. But differential rotation is certainly present and many Yohkoh loops do have such a skew, so how does the Sun counteract it so powerfully in prominences?

We next consider the possibility of effect III. For the Leroy maps we calculated the potential magnetic field from Kitt-Peak magnetograms (van Ballegooijen et al. 1998) and found that all the polar-crown prominences have potential fields that are essentially normal to the polarity inversion line rather than having the large axial component that is required. (For the low-latitude prominences we found that for one-third of the cases the potential field possesses a strong shear, 
most of which have the correct chirality, so for them explanation III works). We then tried hard to avoid a subsurface explanation by taking a realistic source distribution and acting on it with differential rotation, meridional flow and diffusion (van Ballegooijen et al. 1998). The result of this modelling was unsuccessful in that it produced sinistral structures along the northern polar crown.

We were therefore lead naturally to effect II (subsurface evolution) as the only viable mechanism for polar-crown prominences. The simple emergence of a twisted horizontal flux tube from below the photosphere is highly unlikely because the amount of plasma that would be lifted up is too large by a not insignificant factor, namely $10^{9}$ ! Also it is the plasma that dominates the magnetic field below the photosphere for reasonable field strengths and not the reverse. We agree with Rust and Kumar (1995) that initially it is likely that there is a large-scale flux tube below the photosphere and finally, when the prominence has formed, there is a large-scale tube up in the corona, but the key question is what happens in between. How does the magnetic tube become disconnected from the plasma? A plausible answer is given by Dextral-Sinistral Model (Priest et al. 1996) which builds on the earlier ideas of van Ballegooijen and Martens (1989) as follows.

We suggest that differential rotation (or active-region flow) acts on the field below the surface to produce in a natural way the correct direction for the field component along the polarity inversion (i.e., Figure 6a). The field then floats to the surface and emergence and small-scale cancellation builds up the flux of the filament channel along the inversion line with the correct sense (Figure 6b). Next, large-scale flux cancellations form long field lines parallel to the inversion line which lift cool mass up into the corona (Figure 6c). We suggest that the filament consists of many such threads along the filament channel. The net effect is to create a weakly twisted flux tube, left-handed in the northern hemisphere. The twist arises because of the conservation of magnetic helicity during the reconnections. When the filament and arcade erupt they produce a magnetic cloud in interplanetary space with the correct sense of twist.

\subsection{Low-Latitude Prominences}

Now consider other kinds of quiescent prominence. There are two generic cases of typical flux distributions that often occur on the Sun and may give the correct chirality by effect III. First of all, the spread and distortion of active-region flux naturally produces dextral fields in the north, as we have confirmed by detailed studies of the evolution of either a simple bipole or of a Kitt Peak map (van Ballegooijen et al. 1998). Once the correct sign for the axial flux has been produced, it may be built up by convergence towards the polarity inversion line.

A second common case is when a new active region emerges at low latitude next to a remnant active region. The resulting flux distribution at the boundary between the new and old regions is found to have the correct chirality either if the relative convergence is included (Galsgaard and Longbottom 1998, these proceedings), or if the force-free field of the new active region with the appropriate helicity for that hemisphere is present (Mackay et al. 1998). For example, emergence of a new active region with positive $\alpha$ in the southern hemisphere produces a sinistral channel when it emerges to the right of the remnant active region and no channel (i.e., normal flux) when it emerges to the left. 


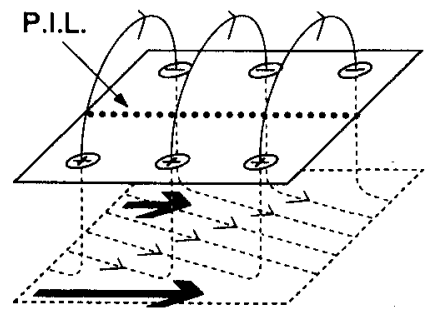

(a) Preparation (dextral)

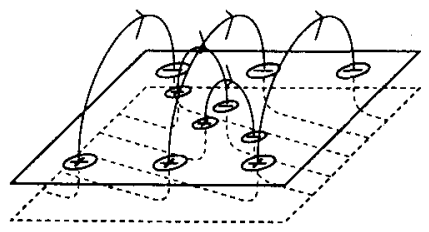

(b) Filament channel formation

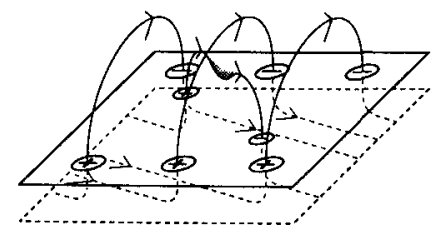

(c) Filament formation

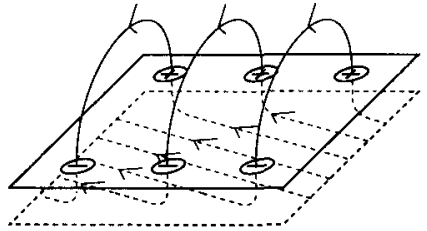

(d) Preparation (dextral)

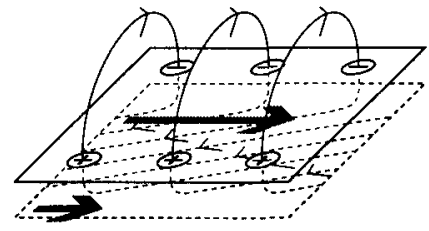

(e) Preparation (sinistral)

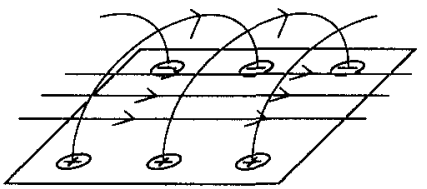

(f) Dextral field before eruption

Figure 6. The dextral-sinistral model for the formation of a filament channel and a filament.

\section{Conclusion}

With helpful suggestions from S. Martin, O. Engvold, T. Forbes, K. Harvey and J. Zirker, the St. Andrews group and A. van Ballegooijen have been trying to set up a coherent model for the prominence phenomena that brings together many diverse observational features and explains them in a natural way. The model has three parts:

(i) an explanation for the global chiral structure of polar-crown prominences in terms of subsurface evolution and of many low-latitude prominences in terms of generic flux distribution;

(ii) the global structure of a prominence and its filament channel, consisting of a flux tube along the channel separated from an overlying arcade by a separatrix surface;

(iii) the local thread-like structure, consisting of plasma that is continually condensing and heating up again and does not need support.

Some of the old paradigms are being replaced in this basic rethinking of the 
fundamentals of prominence physics:

(A) Formation may be due to either the lifting of mass magnetically from the lower atmosphere, or it may be the continual forming by radiative condensation and heating in a dynamic way, rather than a single radiative condensation;

(B) The structure is likely to be a flux tube rather than a sheared arcade; in addition, the organisational principle that creates the chirality, forms the flux tube and supplies the mass is a combination of (i) subsurface flows, emergence and reconnection for polar-crown prominences, and (ii) generic flux distributions and converging flux for many low-latitude prominences;

(C) Prominence material is unlikely to be essentially static; instead we suggest that it is either continually replenished by magnetic reconnection at a rate that balances the natural loss; or dynamically recycled between hot and cool components at a rate that means there is no need for support of the material in its cool phase since it does not have time to drain down;

(D) The structure of the filament channel and the barbs may be a natural consequence of the distribution of magnetic flux sources within the channel.

Acknowledgments. I am delighted to acknowledge support from the UK Particle Physics and Astronomy Research Council and members of the PROM consortium and the St. Andrews Solar Theory Group.

\section{References}

Antiochos, S.K. and Klimchuk, J.A. 1993, ApJ, 370, 372

Aulanier, G. and Démoulin, P. 1998, A\&A, 329, 1125

Engvold, O. 1989, in Dynamics and Structure of Quiescent Solar Prominences, (ed.) E.R. Priest, Kluwer Acad. Publ., Dordrecht, Holland, p. 47

Hood, A. and Priest, E.R. 1979, A\&A, 77, 233

Leroy, J.L. 1989, in Dynamics and Structure of Quiescent Solar Prominences, (ed.) E.R. Priest, Kluwer Acad. Publ., Dordrecht, Holland, p. 77

Low, B.C. 1991, ApJ, 381, 295

Mackay, D. and Priest, E.R. 1996, Solar Phys., 167, 281

Mackay, D., Gaizauskas, V., Rickard, G. and Priest, E.R. 1997, ApJ, 486, 534

Mackay, D. et al. 1998, Solar Phys., in press

Martin, S.F., Bilimoria, R. and Tracadas 1994, Solar Surface Magnetism, R.J. Rutten and C.J. Schrijver (eds.), Springer-Verlag, New York, p. 303

Priest, E.R., Hood, A. and Anzer, U. 1989, ApJ, 344, 1010

Priest, E.R., Van Ballegooijen, A. and Mackay, D. 1996, ApJ, 460, 530

Rust, D.M. and Kumar, A. 1995, Solar Phys., 155, 69

van Ballegooijen, A.A. and Martens, P.C.H. 1989, ApJ, 343, 971

van Ballegooijen, A.A. and Martens, P.C.H. 1990, ApJ, 361, 283

van Ballegooijen, A.A. et al. 1998, ApJ, in press 